\title{
Aortico-Left Ventricular Tunnel: Report of Two Prenatal Cases and Review of the Literature
}

\section{Introduction \\ $\nabla$}

Aortico-left ventricular tunnel (ALVT) is a rare congenital heart malformation representing approximately $0.001 \%$ of all congenital cardiac malformations (Okoroma EO et al. J Thorac Cardiovasc Surg. 1976; 71: 238-44). ALVT was first described as an abnormal communication between the ascending aorta and the left ventricle (Levy MJ et al. Circulation 1963; 27: 841-53). The tunnel begins above the sinus of Valsalva near the right coronary ostium and ends in the left ventricle below the right aortic cusp. The embryological basis is unknown. Diagnosis can be made by fetal echocardiography. Possible features of the lesion during fetal life include a dilated left ventricle, aortic regurgitation and a dilated ascending aorta. Postnatal treatment is early surgical patch closure of the defect. A good longterm outcome postoperatively is reported. However, the outcome is highly dependent on the antenatal presence of heart failure and hydrops (Singh A et al. Pediatr Cardiol 2011; 32: 822-5). The course of an untreated large ALVT is progressive heart failure leading to death. We present two second trimester cases with signs of severe heart failure due to ALVT which were diagnosed by autopsy.

\section{Case Description \\ $\nabla$ \\ Case 1}

A 32-year-old pregnant woman with a normal nuchal scan attended routine malformation scan at 19 weeks +3 days. The heart was found to be enlarged with a dilated left ventricle. The left ventricle was described as being connected to a hypoplastic right ventricle through a large perimembranous ventricular septal defect (VSD). The mitral valve was stenotic with reverse flow through the foramen ovale. The aorta appeared very dilated with high flow velocities in the ascending aorta and severe aortic regurgitation. The parents were informed of the poor prognosis and opted for termination of the pregnancy. They gave permission for autopsy, which showed a male fetus appropriate for gestational age with an ALVT. The left ventricular outflow tract was very dilated with an aneurysmal dilatation ( $\bullet$ Fig. 1b) in the ventricular septum with a volume equal to the left ventricle. The dilatation was located under a tunnel defect to the ascending aorta. The aneurysm was visible upon reassessment of the ultrasonic pictures ( $\bullet$ Fig. 1c). The aortic valve was hypoplastic and the ascending aorta was severely dilated. The left ventricle was dilated and hypertrophic with fibroelastosis and a wall thickness 3 times the thickness of the right ventricle. The right ventricle was hypoplastic. The foramen ovale membrane was thin, consistent with the clinical observation of a left-to-right shunt. No extracardiac malformations were found.

\section{Case 2}

A 35-year-old woman, first pregnancy with a normal nuchal scan. At a routine malformation scan at 20 weeks, the fetus was found dead with dimensions appropriate for 16 weeks. Labor was induced the following day. Autopsy showed a
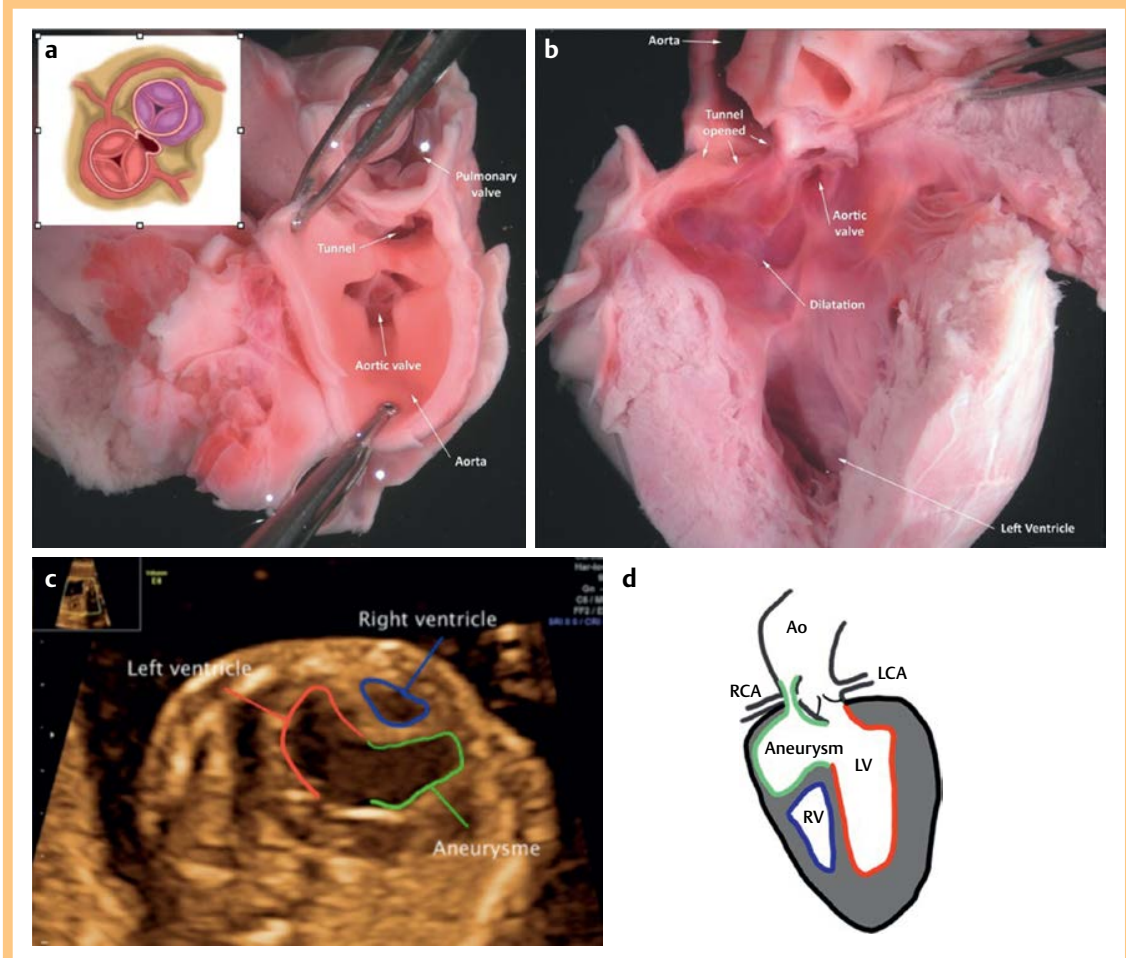

d

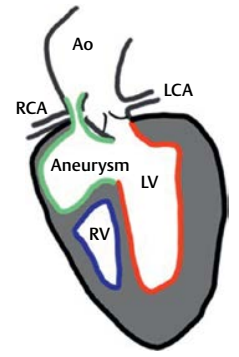

Fig. 1 Case 1: The tunnel defect is seen anterior to the hypoplastic aortic valve a above an aneurismal dilatation of the left ventricular outflow tract b. c Echocardiographic image of the left ventricle, right ventricle and the left ventricle aneurysm. Before the autopsy, the aneurysmal dilatation was misinterpreted as being part of the right ventricle, and thus there was an impression of the presence of a large VSD. $\mathbf{d}$ Schematic illustration. 


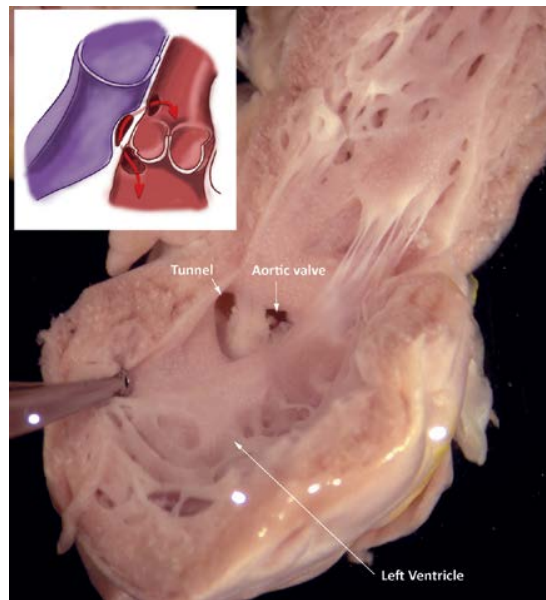

Fig. 2 Case 2: Left ventricle opened. The ventricular wall is thick. Anterior to the hypoplastic aortic valve, a tunnel defect is present connecting the left ventricle with the ascending aorta.

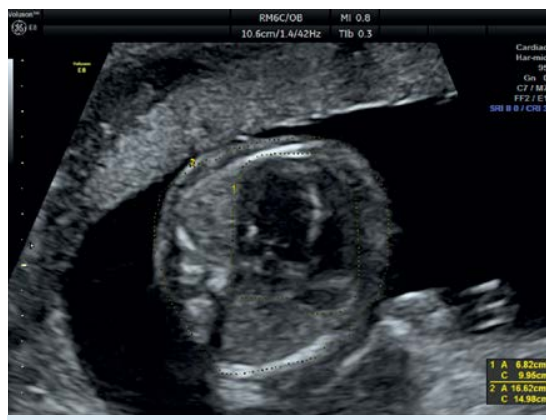

Fig. 3 Four-chamber view.

male fetus with severe maceration; appropriate for 15 weeks. The heart was markedly hypertrophic, and the weight was appropriate for 19 weeks. The left ventricle was enlarged, rounded and thick-walled. There were broadened trabeculae and fibroelastosis. The outflow tract showed 2 outlets, posteriorly a hypoplastic aortic valve, and anteriorly an abnormal separate tunnel-shaped opening connecting the left ventricle with the ascending aorta. The distal aortic arch was hypoplastic. ALVT was most probably the cause of the intrauterine fetal death - Fig. 2.

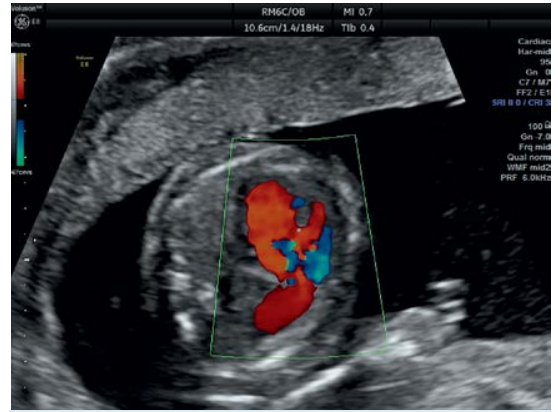

Fig. 4 Color Doppler findings from aorta and tunnel.

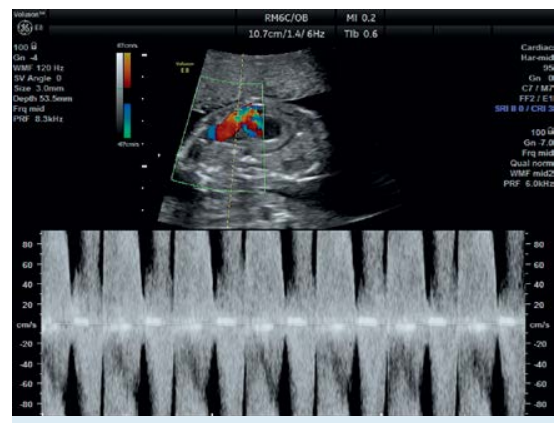

Fig. 5 Doppler waveforms from aorta and tunnel.

\section{Discussion}

$\nabla$

ALVT is a well-described condition in children and may be diagnosed prenatally. Before our publication, the tunnel has been reported in approximately 100 cases. Only 16 cases (Singh A et al. Pediatr Cardiol 2011; 32: 822-5; Sousa-Uva M et al. Ann Thorac Surg 1996; 61: 1805-10) were diagnosed prenatally, of which 5 children survived after postnatal surgery. The main feature of ALVT is aortic regurgitation. The lesion might be suspected in the four-chamber view due to a dilated left ventricle. Color flow mapping may demonstrate turbulent regurgitant flow that seems to flow across the aortic valve but is upon closer inspection around the valve (Allan L et al. Textbook of Fetal Cardiology. Greenwich Medical Media 2000: 229-32). Additional signs are aortic root dilatation, causing narrowing of the right ventricular outflow tract, and thickening and stenosis of the aortic valve, causing left ventricle dilatation, all of which were present in our 2 cases $\bullet$ Fig. 3-5.

The age of presentation and severity of symptoms depends on the size of the tunnel. Case 1 illustrates that an ALVT may clinically mimic aortic regurgitation as well as absent aortic valve syndrome. A VSD was suspected ultrasonically but not confirmed by autopsy. The appearance of the dilated left ventricular outflow tract can sometimes be confused with a large VSD (Allan L et al. Textbook of Fetal Cardiology. Greenwich Medical Media 2000: 229-32). Diastolic reversal aortic flow suggests severe tunnel regurgitation, as was seen in case 1 . It is likely that the ALVT cases detected in utero represent the worst end of the symptom spectrum. Most of the reported fetal cases presented in the second trimester as our 2 cases. Case 2 resulted in missed abortion. The fetus showed severe cardiac hypertrophy and would probably have shown signs of hydrops, had it not been so macerated. The milder forms seen postnatally, with only mild or moderate left ventricular dysfunction and without any major aortic valve abnormalities, are likely to go undetected in fetal life (Sousa-Uva M et al. Ann Thorac Surg 1996; 61: 1805-10). A thorough Doppler examination of the left ventricular outflow tract will demonstrate the forward flow into the aorta and the reversed flow into the left ventricle through the tunnel. Our report exemplifies how an ALVT may be misdiagnosed even by experienced examiners most likely because it is so rare. The diagnostic clue is to consider ALVT when regurgitant aortic flow is seen. Prenatal diagnosis allows a postnatal treatment plan for the fetus or the opportunity of abortion in severe cases.

I. K. Greiber, L. L. Maroun, N. VejIstrup, F. S. Jørgensen; Copenhagen 